\title{
Perfil sociodemográfico y actitudes políticas de los grupos a favor y en contra de limitar la libre circulación de información durante la pandemia
}

\author{
Socio-demographic profile and political attitudes of \\ groups for and against limiting the free circulation of \\ information during the pandemic
}

\author{
Lidia Valera-Ordaz; Jaume Doménech-Beltrán
}

Cómo citar este artículo:

Valera-Ordaz, Lidia; Doménech-Beltrán, Jaume (2020). “Perfil sociodemográfico y actitudes políticas de los grupos a favor y en contra de limitar la libre circulación de información durante la pandemia". Profesional de la información, v. 29, n. 6, e290616.

https://doi.org/10.3145/epi.2020.nov.16

Artículo recibido el 11-08-2020 Aceptación definitiva: 21-10-2020

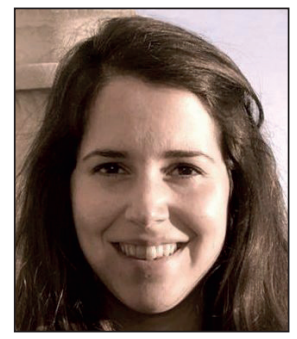

Lidia Valera-Ordaz $\bowtie$ https://orcid.org/0000-0002-1085-980X

Universidad de Valencia

Dpto. Teoría de los Lenguajes y CC. de la Comunicación

Avda. Blasco Ibáñez, 32

46010 Valencia, España

lidia.valera.ordaz@gmail.com

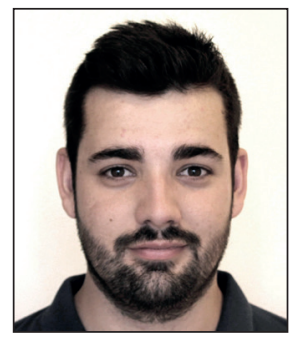

Jaume Doménech-Beltrán

https://orcid.org/0000-0002-4837-6811

Universidad de Valencia

Dpto. Teoría de los Lenguajes y CC. de la Comunicación

Avda. Blasco Ibáñez, 32

46010 Valencia, España

jaudobel@alumni.uv.es

\section{Resumen}

La pandemia ocasionada por la Covid-19 ha supuesto no sólo una terrible crisis sanitaria, sino importantes problemas relacionados con la difusión de desinformación en el contexto de sistemas mediáticos híbridos y crecientemente fragmentados. Analizamos la polémica pregunta del CIS (Centro de Investigaciones Sociológicas) sobre la pertinencia de limitar la libre circulación de información para restringir la difusión de bulos y noticias falsas. El objetivo es: 1) analizar las características sociodemográficas básicas de quienes se declararon partidarios de restringir la libre circulación informativa, y 2) explorar sus inclinaciones políticas e ideológicas, y su asociación con las actitudes en torno a la libertad informativa. Así, mediante una metodología cuantitativa basada en tablas de contingencia y residuos tipificados corregidos, los resultados reflejan que el perfil sociodemográfico más frecuente entre los partidarios de limitar la libertad informativa es el de una mujer joven (entre 18 y 25 años), con estudios secundarios, que vive en municipios pequeños y declara pertenecer a la clase social baja. Además, los resultados ilustran que existe una asociación estadística significativa entre apoyar la restricción informativa y diversos indicadores de apoyo al Gobierno central: votar y simpatizar con el PSOE, situarse en la extrema izquierda, confiar en la gestión de la pandemia del Gobierno central y confiar en su presidente.

\section{Palabras clave}

Libertad de información; Restricción del flujo informativo; Perfiles sociodemográficos; Identificación partidista; Sociología; Partidos políticos; Desinformación; Noticias falsas; Crisis sanitarias; Pandemias; Covid-19; Coronavirus; Encuestas; CIS.

\section{Financiación}

Este trabajo se enmarca en el proyecto de investigación "Ecología de la desinformación: la construcción de las noticias falsas y su impacto en el espacio público” (AICO2020/224), dirigido por Guillermo López-García, y financiado por la Generalitat Valenciana (2020-2021). 


\begin{abstract}
The Covid-19 pandemic has produced not only a terrible sanitary crisis but also several problems related to the circulation of disinformation in the context of hybrid and increasingly fragmented media systems. In this work, we analyze a polemical question included in the Center for Sociological Research (CIS) survey conducted in April about the appropriateness of limiting free circulation of information to avoid diffusion of fake news and disinformation. The goals are to (1) analyze the sociodemographic traits of those in favor of limiting the free circulation of information, and (2) explore their general political preferences and affiliations, and the association with attitudes regarding freedom of information. The results obtained through a quantitative methodological approach based on contingency tables and standardized residuals indicate that the most common sociodemographic profile of those in favor of limiting the flow of information is the following: young women (between 18 and 25 years) with secondary education who live in small municipalities and belong to the lower social class. Moreover, the findings illustrate that there is a significant statistical association between supporting the limitation of information and different indicators of supporting the Socialist government: voting and sympathizing with the Socialist Party, self-location in the extreme left, and trusting both the central government management of the crisis and the leadership of Pedro Sánchez.
\end{abstract}

\title{
Keywords
}

Free circulation of information; Limitation of information flows; Sociodemographic profiles; Partisan identification; Sociology; Disinformation; Fake news; Health crisis; Covid-19; Pandemics; Coronavirus; Surveys; CIS.

\section{Introducción}

\subsection{Crisis sanitaria de la Covid-19}

El 31 de enero de 2020 España reportó su primer caso de Covid-19: un turista alemán había dado positivo en La Gomera. No fue hasta el 14 de marzo, con 5.232 contagiados y 133 muertos, cuando el Gobierno español decidió decretar el Estado de alarma y confinar a la población, a pesar de que un brote en la región italiana de Lombardía había precipitado el confinamiento de esa región unas semanas antes. Una vez la gravedad de la situación era manifiesta, la oposición centró sus acusaciones contra el Ejecutivo en la manifestación del Día Internacional de la Mujer. El Partido Popular y Vox acusaron al Gobierno de irresponsabilidad al mantener la movilización a pesar de los riesgos de contagio. Por su parte, el Gobierno de coalición se defendía arguyendo que todas sus decisiones estaban basadas en las recomendaciones ofrecidas por los expertos sanitarios.

Apenas seis días después de la celebración de la manifestación del 8 de marzo, el presidente del Ejecutivo, Pedro Sánchez, anunciaba el decreto por el que se aprobaba el Estado de alarma. El decreto supuso una restricción sin precedentes de derechos y libertades en la historia de la democracia española, que no obstante la mayoría de la ciudadanía aceptó como medida imprescindible para contener los devastadores efectos de la pandemia (Amat et al., 2020). A este primer anuncio del presidente del Gobierno le seguirían sucesivas extensiones del Estado de alarma, dos meses de confinamiento, y el inicio de una crisis de grandes proporciones, que incluiría el colapso del sistema sanitario hacia finales del mes de marzo.

Desde la aprobación del Estado de alarma, la estrategia de comunicación del Gobierno español ante la crisis se basó en cuatro ejes fundamentales (López-García, 2020):

- comunicación continua mediante ruedas de prensa televisadas, que se celebraban en horarios de máxima audiencia;

- justificación de la restricción de los derechos y libertades de los ciudadanos atendiendo a la gravedad de la crisis;

- búsqueda de una imagen de control de la situación;

- apelación a la unidad.

El discurso gubernamental apeló sistemáticamente a la unión de la ciudadanía y las fuerzas políticas, mientras que trataba de trasladar una imagen de liderazgo y solvencia en la respuesta a la crisis. Esta apelación a la unidad se manifestó en la relevancia otorgada a las Fuerzas Armadas, Guardia Civil y Policía Nacional, que aparecían de manera habitual en las ruedas de prensa en un intento claro del Gobierno de capitalizar su buena imagen pública ante la ciudadanía (López-García, 2020).

A nivel europeo, estudios recientes subrayan también la falta de una estrategia de comunicación integrada entre los líderes de los países europeos más golpeados, y un uso significativamente variado de Twitter durante los primeros meses de la pandemia (Drylie-Carey; Sánchez-Castillo; Galán-Cubillo, 2020). En relación con la cobertura mediática de la pandemia, algunas investigaciones advierten que la televisión pública jugó un papel importante en la percepción del riesgo, de forma que durante los meses de enero y febrero la cobertura se centró en una amenaza lejana situada en el extranjero y a partir de marzo el volumen de información creció notablemente y acabó por ser excesivo, de acuerdo con las percepciones de la audiencia (Villena-Alarcón; Caballero-Galeote, 2020). 


\subsection{La esfera pública española}

El sistema mediático español es un claro ejemplo del modelo de pluralismo polarizado (Hallin; Mancini, 2004), propio de los países del Sur de Europa, con un nivel relativamente alto de paralelismo político. Los medios de comunicación tienden a estar politizados de forma que los periódicos, los canales de televisión y las emisoras de radio representan posturas ideológicas bien definidas, que a su vez están alineadas con los partidos que conforman el sistema de partidos español. Ello se manifiesta tanto en los temas que los medios privilegian en su cobertura (Valera-Ordaz, 2015), como en los encuadres partidistas que reproducen (Valera-Ordaz, 2019; Carratalá; Valera-Ordaz, 2020). En los sistemas pluralistas polarizados los periodistas tienden a exhibir un estilo periodístico más próximo al comentario que a la información, lo que sin duda contribuye a dar forma a una esfera pública en la que el ataque al rival político es habitual.

Por otra parte, los sistemas mediáticos han experimentado una intensa transformación con motivo de la digitalización. Algunos autores definen la actual coexistencia de medios, contenidos y formatos como "sistemas mediáticos híbridos" (Chadwick, 2013). La expansión de la oferta derivada de la digitalización y la significativa fragmentación del consumo, junto con la quiebra de los modelos de negocio tradicionales, han aumentado los incentivos para que los medios se concentren de forma creciente en nichos ideológicos específicos de audiencia, tratando así de fidelizar un segmento del mercado (Bennett; lyengar, 2008; Mullainathan; Shleifer, 2005) en un contexto de incertidumbre, disminución de los ingresos por publicidad y aumento de la competencia. Esto ha sucedido no sólo en los sistemas mediáticos con audiencias históricamente partidistas (Humanes, 2014), como el español, sino también en otros en los que la prensa comercial y la neutralidad política de los grandes medios estaban más firmemente arraigados, cuyo ejemplo paradigmático sería el mercado estadounidense de la televisión generalista (Stroud, 2010).

La fragmentación y la polarización también han afectado a los ecosistemas multimedia, de forma que en la esfera pública digital, las audiencias se inclinan por el consumo de información alineada con sus actitudes políticas (Van-Aelst et al., 2017). Los usuarios en general prefieren la información congruente con sus propios valores y actitudes políticas, con tal de ahorrarse el esfuerzo cognitivo que supone cuestionar sistemáticamente actitudes previamente formadas (Stroud, 2011). Así lo constatan numerosos estudios empíricos que se inscriben en la corriente de investigación de la teoría de la exposición selectiva, tanto en España como en otros países (Stroud, 2008; 2010; Humanes, 2014; Humanes; Mellado, 2017; Bos; Kruikemeier; De-Vreese, 2016; Goldman; Mutz, 2011). Todos estos estudios constatan empíricamente que la ideología y la identificación partidista son los mejores predictores de la dieta mediática de los individuos en diversos entornos sociopolíticos.

En este escenario no resulta sorprendente que la desinformación, los rumores y las teorías de la conspiración penetren con facilidad en el discurso mediático (Aleixandre-Benavent; Castelló-Cogollos; Valderrama-Zurián, 2020) -muchas veces vía redes sociales-, y que la información fáctica tienda a ser sistemáticamente cuestionada y equiparada con enunciados de opinión (Graves, 2017; Van-Aelst et al., 2017). El sesgo de selección es susceptible también de afectar al denominado periodismo de verificación o fact-checking, en ascendencia en los últimos años en España desde su origen estadounidense (López-Pan; Rodríguez-Rodríguez, 2020), ya que los periodistas dedicados a la verificación de noticias pueden fácilmente evitar desacreditar informaciones alineadas con sus actitudes políticas, y desmentir en mayor medida las noticias falsas más contrarias a sus valores (Hameleers; Van-der-Meer, 2019). Algo similar sucede con los ciudadanos, que tienden a compartir en redes sociales en mucha mayor medida los mensajes de verificación que dejan en buen lugar a sus candidatos políticos preferidos o que desmienten al rival ideológico (Shin; Thorson, 2017). Especialmente en sistemas mediáticos como el español, en los que los periodistas tienen una fuerte vocación de movilización social y se ven a sí mismos como agentes comprometidos con valores políticos, en comparación con sus colegas europeos (Van-Dalen; De-Vreese; Albæk, 2012). Un estudio reciente señala por ejemplo, que el debate en Twitter durante las elecciones generales de abril de 2019 estuvo dominado por el live fact-checking que hacían los medios de comunicación de izquierda, que se dedicaban a desmentir al candidato popular Pablo Casado (Paniagua-Rojano; Seoane-Pérez; Magallón-Rosa, 2020).

Este incremento de la fragmentación del consumo y la polarización política en el entorno digital pueden tener consecuencias nocivas que van más allá del relativismo y el cuestionamiento del discurso factual. De hecho, la fragmentación del consumo mediático alienta lo que algunos han llamado la "sentimentalización de la conversación pública" -convenientemente encapsulada en nichos ideológicos-y el auge de las identidades grupales de naturaleza afectiva (Arias-Maldonado, 2016). Con ello no sólo se erosiona la legitimidad de los medios de comunicación como actores que producen información contrastada y simultáneamente sirven de foros públicos para acoger el debate en torno a los asuntos de interés general. También se socava el discurso argumentativo, creando un clima favorable a la diseminación de prejuicios y emociones exaltadas, propiciando en última instancia el auge de la intolerancia hacia la discrepancia ideológica, y erosionando la cohesión social (Van-Aelst et al., 2017).

La pandemia ha estado acompañada de una eclosión de teorías de la conspiración, tesis negacionistas, noticias falsas y una gran ola de desinformación 


\subsection{Debate en torno a la desinformación y las noticias falsas}

Con este trasfondo, la pandemia ha estado acompañada de una eclosión de teorías de la conspiración, tesis negacionistas, noticias falsas, y una gran ola de desinformación a través de los medios digitales y las redes sociales -supuestos remedios contra el virus, fórmulas variopintas de prevención, teorías conspiranoicas, etc.-. Las informaciones erróneas sobre la Covid-19 han sido habituales e incluso en ocasiones han sido difundidas por mandatarios políticos como Jair Bolsonaro en Brasil o Donald Trump en Estados Unidos. Todo ello ha facilitado no sólo la circulación de noticias falsas en redes sociales, sino también su salto a los contenidos periodísticos, que se hacían eco de las tesis negacionistas y acientíficas más populares de Internet.

Las redes sociales alientan la rápida difusión de las noticias falsas, pues carecen de mecanismos eficaces de verificación y por lo tanto permiten que se publique información sin cumplir con criterios mínimos de veracidad. Diversos autores advierten que la consolidación de las redes sociales como canales de comunicación supone un problema grave de desintermediación, en tanto que erosiona el papel de los grandes medios en la esfera pública como intérpretes privilegiados del mundo social y político (Masip; Ruiz-Caballero; Suau, 2019; Fisher et al., 2019; Broersma; Graham, 2013). Esta desintermediación facilita que las noticias falsas prosperen, porque la información puede viajar desde el emisor de contenido hasta la audiencia sin pasar ningún filtro interpretativo (Cooke, 2018), dejando además a la información prisionera del marco de interpretación de su emisor interesado.

Los riesgos que tiene la desinformación para la democracia son unánimemente reconocidos por organizaciones internacionales como la Comisión Europea y la ONU, que coinciden en la necesidad de combatirlos para proteger la calidad del debate público y, en última instancia, evitar la perversión del derecho a la libertad de información. Así lo recogen por ejemplo documentos como

- el Código de prácticas de la Unión Europea en materia de desinformación (European Commission, 2018b);

- la Comunicación de la Comisión al Parlamento Europeo, al Consejo, al Comité Económico y Social Europeo y al Comité de las Regiones "La lucha contra la desinformación: un enfoque europeo" (European Commission, 2018a);

- la Declaración conjunta sobre la libertad de expresión y noticias falsas, desinformación y propaganda (ONU, 2017), adoptada por los relatores especiales para la libertad de opinión y de expresión de la ONU, la OSCE, la OEA y la CADHP.

El problema es que legislar sobre el fenómeno de la desinformación no resulta jurídicamente sencillo, como tampoco lo es hacer cumplir la legislación en torno a los límites que ya existen sobre la libertad de información. De hecho, entraña riesgos nada desdeñables como la censura. Así lo recoge por ejemplo la Declaración conjunta sobre la libertad de expresión y noticias falsas, desinformación y propaganda (ONU, 2017), que recomienda lo siguiente:

"las prohibiciones generales de difusión de información basadas en conceptos imprecisos y ambiguos, incluidos 'noticias falsas' ('fake news') o 'información no objetiva', son incompatibles con los estándares internacionales sobre restricciones a la libertad de expresión, conforme se indica en el párrafo 1(a), y deberían ser derogadas" (ONU, 2017).

Pero además la facilidad para producir y consumir noticias falsas se ha agravado con la pandemia, que ha precipitado el aumento de la demanda de información y un uso intensivo de múltiples canales de comunicación (Moreno; FuentesLara; Navarro, 2020). El alto nivel de incertidumbre y la necesidad de estar informado constantemente han afectado probablemente al filtro del consumidor, que quizás ha dado por válidas informaciones que en un contexto de normalidad habría cuestionado o juzgado como erróneas, falaces o poco rigurosas. Asimismo, la difusión de fake news e informaciones poco contrastadas resulta particularmente perjudicial en un escenario de crisis sanitaria, ya que conduce al temor excesivo, al desconocimiento de la gravedad de la situación, a la inobservancia de las directrices sanitarias, y/o al uso de tratamientos perjudiciales para la salud (Rosenberg; Syed; Rezaie, 2020).

No obstante, conviene también poner de manifiesto que la falta de información científica consensuada entre epidemiólogos y expertos, unida a la enorme demanda de información por parte de los ciudadanos y a los sucesivos y en ocasiones inexplicables cambios de criterio por parte de las autoridades sanitarias, científicas y políticas (cuyo ejemplo paradigmático sería la pertinencia del uso de mascarillas), han creado un ambiente bastante propicio para la circulación de toda clase de información, en el mejor de los casos imprecisa, y en el peor, rotundamente falsa.

Un estudio reciente de Casero-Ripollés (2020, p. 9) muestra que la pandemia ha causado un espectacular aumento de consumo de información en Estados Unidos, lo que subraya que la información veraz se ha convertido en un valiosísimo recurso para los ciudadanos en situaciones de emergencia. El aumento ha sido tan significativo que ha reducido de forma importante las tradicionales desigualdades en el consumo de información entre diferentes grupos sociodemográficos, mitigando el impacto de las variables como la educación, el estatus socioeconómico y la edad sobre el consumo mediático, y reconectando con la actualidad a sectores demográficos tradicionalmente alejados del consumo de noticias (jóvenes, individuos sin estudios, etc.). Asimismo, el estudio subraya que los medios de comunicación tradicionales han sido las fuentes preferenciales de información para la población estadounidense -frente a los medios sociales- pues eran percibidas como más creíbles y confiables (Casero-Ripollés, 2020).

La gran demanda de información contrastada y veraz, junto con la circulación de información falsa e inexacta, ha propiciado en el caso español la politización de la cuestión de las noticias falsas, que ha sido utilizada como estrategia de 
confrontación política entre los partidos. El casus belli más destacado tiene que ver con el número de fallecidos, que ha sido motivo de sucesivos reproches por parte de la oposición y de confrontación permanente entre el Gobierno y el resto de las fuerzas políticas. La oposición empezó a reprochar al Gobierno no ofrecer datos exactos de fallecidos por Covid-19 -pues sólo se incluían en los datos oficiales los fallecidos con diagnóstico realizado mediante prueba PCR y no todos los que habían muerto con sintomatología compatible con el virus, como sugería la Organización Mundial de la Salud-. Por su parte, el Ejecutivo acusaba a las fuerzas de la oposición de deslealtad (especialmente cuando los partidos de la derecha cuestionaron su apoyo parlamentario para prolongar el Estado de alarma), de querer desestabilizar al Gobierno y de capitalizar políticamente la crisis sanitaria. Además, las críticas a la gestión gubernamental de la crisis sanitaria, que tuvieron primero una fuerte presencia en las redes sociales, y posteriormente se trasladaron a la calle cuando las medidas comenzaron a relajarse, fueron tildadas por el Gobierno como una campaña de bulos y desinformación organizada por los partidos políticos de la derecha.

A estas acusaciones cruzadas entre partidos se han unido también los medios de comunicación, que han centrado su cobertura en atacar la gestión de las administraciones públicas gobernadas por sus enemigos ideológicos naturales. Así, los medios alineados con la izquierda han tenido como diana preferente el Gobierno de la Comunidad de Madrid de Isabel Díaz Ayuso, mientras que los medios de centro y derecha se han cebado con el Gobierno central y su gestión, centrando sus ataques en el PSOE o Podemos, alternativamente.

\subsection{La pregunta de la discordia}

En este contexto de cruce de acusaciones en torno a los bulos, el Centro de Investigaciones Sociológicas (CIS) realizó un barómetro durante los últimos días de marzo y primeros de abril de 2020 -externalizado mediante la contratación de una empresa que realizó entrevistas telefónicas, dada la situación-, e incluyó una pregunta que suscitaría bastante polémica tanto por su formulación como por su resultado. La pregunta 6 del estudio n. 3279, responsable de la controversia, decía:

“¿Cree Ud. que en estos momentos habría que prohibir la difusión de bulos e informaciones engañosas y poco fundamentadas por las redes y los medios de comunicación social, remitiendo toda la información sobre la pandemia a fuentes oficiales, o cree que hay que mantener libertad total para la difusión de noticias e informaciones?".

La formulación de la pregunta recibió numerosas críticas debido a su larga extensión, y a su falta de claridad. También se especuló sobre los motivos que llevaron al CIS a incluirla en el barómetro y formularla de esta forma en particular. Algunos afirmaron que su formulación buscaba claramente inducir la respuesta de los encuestados hacia la opción más coercitiva y, con ello, legitimar la posición del Gobierno y sus declaraciones en torno a la pertinencia de restringir y castigar la circulación de bulos. La acusación se sustentaba en la conocida afiliación socialista del director del CIS, José Félix Tezanos, y su polémica presidencia del CIS. Al mismo tiempo, otros vieron en el elevado porcentaje favorable al control de las informaciones una respuesta a la situación de excepcionalidad e incertidumbre, de forma que la predisposición a la prohibición estaba motivada por la gran demanda de información y el miedo a la desinformación.

Merece la pena destacar que la pregunta incluía simultáneamente cuatro tipos de contenidos susceptibles de ser prohibidos:

- bulos;

- informaciones engañosas;

- informaciones poco fundamentadas;

- informaciones provenientes de fuentes no oficiales.

En lugar de realizar cuatro preguntas distintas que pudieran recoger la variabilidad de actitudes de la población española sobre la pertinencia de restringir la difusión de cada tipo de contenido, el organismo optó por realizar una única pregunta que incluyera los cuatro tipos de fuentes simultáneamente.

Esta decisión metodológica hace que resulte imposible diferenciar entre quienes quizá apoyaban la prohibición de algunos contenidos, pero no de otros. Conviene también subrayar que en principio la protección constitucional de la libertad de información ampara totalmente la publicación de información poco fundamentada, así como la que procede de fuentes no oficiales, pero no necesariamente resulta equiparable con el derecho a difundir información engañosa y bulos (España, 1978, art. 20.1.d). En cualquier caso, teniendo en cuenta que la pregunta del CIS incluyó en su formulación 
estas cuatro categorías de contenidos, parece claro que ésta trataba de registrar precisamente la opinión de los encuestados sobre diferentes fórmulas de restricción de la libertad de información.

¿Pero cuáles fueron las respuestas de los ciudadanos a la pregunta? La tabla 1 recoge las respuestas de los encuestados. El 66,7\% de los encuestados declaró que había que restringir y controlar las informaciones, mientras que sólo un 30,8\% de los individuos opinaron que no debía prohibirse ni restringirse ningún tipo de información. Es decir, dos tercios de la población estaban a favor de prohibir la difusión de bulos y noticias falsas - pese a que éste ni era un problema nuevo y ni se había problematizado hasta entonces en términos de prohibiciones- y sólo un tercio de la población española era partidaria de mantener la libertad de información intacta pese a las circunstancias excepcionales de la crisis sanitaria.

Tabla 1. Opinión sobre la prohibición de la difusión de bulos y noticias falsas ( $N=3.000)$

\begin{tabular}{|l|r|}
\cline { 2 - 3 } \multicolumn{1}{c|}{} & Frecuencia \\
\hline Cree que habría que restringir y controlar las informaciones & 2.000 \\
\hline Cree que no debe restringirse ni prohibirse ningún tipo de información & 925 \\
\hline No lo sabe, duda & 61 \\
\hline N.C. & 30,8 \\
\hline Total & 2,0 \\
\hline
\end{tabular}

Fuente: Estudio CIS n. 3279

A tenor de estos llamativos resultados -y más allá del posible efecto de la formulación de la pregunta sobre las respuestas-, podríamos decir que la población española experimentó una notable transformación en un período de tiempo significativamente breve, y que la situación de la pandemia afectó de forma intensa a las actitudes en torno a la libertad de información. De forma similar a lo que ocurrió en 1931, cuando el almirante Aznar declaró que "España se acostó monárquica y se levantó republicana" podríamos decir, emulando la célebre frase, que "España se acostó liberal y se levantó prohibicionista".

\section{Objetivos de investigación}

En este trabajo nos proponemos tres objetivos de investigación, que giran en torno a identificar las características de quienes se declararon partidarios de limitar el derecho a la información, y compararlas con quienes mantuvieron actitudes no prohibicionistas a pesar de las circunstancias extraordinarias.

01. Analizar las características sociodemográficas básicas de quienes se declararon partidarios de limitar la libertad de información durante el Estado de alarma, en lo que se refiere al sexo, la edad, el nivel educativo, la clase social, y el tamaño de municipio.

O2. Analizar las actitudes políticas y el perfil ideológico de quienes declararon estar a favor de limitar el control del flujo informativo, en lo relativo al comportamiento electoral, la ideología, la simpatía por partidos políticos, y la confianza en el Gobierno socialista y en el presidente del Gobierno.

O3. Explorar si existe asociación estadística entre las actitudes políticas favorables al PSOE y al Gobierno de coalición, y las actitudes en torno a la restricción del flujo informativo.

\section{Metodología}

Para cartografiar el perfil sociodemográfico e ideológico de los partidarios de la prohibición de la libre circulación de la información durante la crisis sanitaria provocada por la Covid-19, hemos utilizado la polémica encuesta postelectoral del CIS n. 3279, administrada telefónicamente a 3.000 individuos mayores de 18 años entre el 30 de marzo y el 7 abril de 2020, durante el período de confinamiento.

Merece la pena subrayar que esta encuesta, a diferencia de todos los cuestionarios que administra el CIS, fue encomendada a la empresa Intercampo S.A. y administrada por vía telefónica a los encuestados mediante una selección aleatoria de teléfonos fijos y móviles y mediante cuotas de sexo y edad, dada la imposibilidad de realizar las entrevistas de forma presencial mediante el procedimiento usual de muestreo que utiliza el CIS. Se trata por lo tanto de un procedimiento extraordinario derivado de las circunstancias, y alejado del procedimiento de muestreo habitual, que consiste en la selección de las unidades primarias de muestreo (municipios) y de las unidades secundarias (secciones) de forma aleatoria proporcional, y de las unidades últimas (individuos) por rutas aleatorias y cuotas de sexo y edad.

Con el fin de responder a los objetivos de investigación, planteamos una exploración bivariable, mediante tablas de contingencia, que permita describir cuáles son las características sociodemográficas y las actitudes políticas generales de quienes fueron partidarios de la restricción de la información durante el Estado de alarma decretado por el Gobierno frente a la población general. Se trata en definitiva de descubrir si el perfil ideológico y sociodemográfico de esta subpoblación es esencialmente el mismo que el de la población general o si, por el contrario, muestra características sustancialmente distintas. 
Además, con el fin de establecer si las diferencias halladas en lo relativo al perfil sociodemográfico y político entre las dos subpoblaciones de interés -las personas partidarias de la prohibición frente a quienes defendían mantener la libertad de información- son significativas, realizamos un análisis de asociación entre las variables mediante la prueba del Chi-cuadrado. Concretamente, utilizamos los residuos tipificados corregidos, que nos permiten observar no sólo si existe asociación estadística entre las variables de interés, sino también observar en qué categorías específicas se concentra dicha asociación mediante valores críticos normalizados.

\section{Resultados}

A continuación, se exponen las tablas de contingencia derivadas de cruzar las actitudes hacia la prohibición de la circulación de información con diversas variables de interés. Estas incluyen atributos sociodemográficos básicos (sexo, edad, nivel de estudios, clase social, tamaño de municipio), y actitudes políticas (ideología, recuerdo de voto, simpatía hacia partidos, confianza en el Gobierno socialista y en el presidente del Gobierno).

Las tablas incluyen los porcentajes de columna y los residuos tipificados corregidos, que cabe interpretar de la siguiente forma: cuando los residuos son mayores (o menores) del valor crítico $+1,96(-1,96)$, existe asociación entre las variables representadas en las tablas al $95 \%$ de probabilidad. Lo mismo sucede con el valor crítico $+2,58(-2,58)$, pero con el $99 \%$ de probabilidad. Es importante enfatizar que cuanto mayor es la magnitud de los residuos, independientemente del signo, más importante es la asociación entre variables en cuestión (la relación entre ellas es más fuerte).

En cada tabla se incluye la muestra total $(\mathrm{N})$ que se corresponde con el cruce de ambas variables, pues algunos casos de análisis se han perdido al realizar los cruces (como las categorías de "No sabe" y "No contesta”, por ejemplo). En muchos casos, además, las variables de interés han sido recodificadas para facilitar los cálculos estadísticos y la interpretación sustantiva de los resultados. Por ejemplo, la variable de edad ha sido recodificada en grupos de edad para poder construir la tabla de contingencia, y en la variable clase social, se han agrupado todas las categorías que incluía el cuestionario en tres grupos: alta, media y baja.

En el caso de la ideología, que se recoge mediante una escala numérica de 10 puntos, se ha recodificado en una nueva variable que recoge cinco grandes orientaciones ideológicas: extrema izquierda (1-2), izquierda (3-4), centro (5-6), derecha (78) y extrema derecha (9-10). Las variables recuerdo de voto y simpatía hacia partidos sólo incluyen a las principales fuerzas políticas estatales ( $P P, P S O E, U P, C S$ y Vox), pues son quienes concentran el grueso del apoyo electoral, y para el resto de los partidos, hay mucha menos muestra de análisis disponible para los cálculos estadísticos, lo que resta validez a los análisis.

Tabla 2. Actitudes hacia la prohibición de información según sexo ( $N=2.986)$

\begin{tabular}{|c|c|c|c|c|}
\hline & & Hombre & Mujer & \\
\hline \multirow{2}{*}{ Habría que restringir y controlar las informaciones } & & $63,4 \%$ & $70,4 \%$ & $67,0 \%$ \\
\hline & Residuo corregido & $-4,1$ & 4,1 & \\
\hline \multirow{2}{*}{ No debe prohibirse ningún tipo de información } & & $34,6 \%$ & $27,6 \%$ & $31,0 \%$ \\
\hline & Residuo corregido & 4,1 & $-4,1$ & \\
\hline \multirow{2}{*}{ No lo sabe } & & $2,1 \%$ & $2,0 \%$ & $2,0 \%$ \\
\hline & Residuo corregido & 0,1 & $-0,1$ & \\
\hline Total & & $100 \%$ & $100 \%$ & $100 \%$ \\
\hline
\end{tabular}

Fuente: Estudio CIS n. 3279

La tabla 2 incluye las actitudes hacia la prohibición de información según el sexo de la población, e indica con claridad que los partidarios de restringir las informaciones fueron en mayor medida mujeres. Así, mientras que un $63,4 \%$ de los hombres se declaraban a favor de la prohibición, el 70,4\% de las mujeres hacía lo mismo. Los residuos tipificados corregidos indican asimismo que estas diferencias son estadísticamente significativas, es decir, que no se deben al azar, y que ambas variables están asociadas al 99\% de probabilidad.

Tabla 3. Actitudes hacia la prohibición de información por grupos de edad ( $\mathrm{N}=2.986)$

\begin{tabular}{|c|c|c|c|c|c|c|c|c|}
\hline & $18-25$ & $26-35$ & $36-45$ & $46-55$ & $56-65$ & $>66$ & \\
\hline \multirow{2}{*}{$\begin{array}{l}\text { Habría que restringir y controlar } \\
\text { las informaciones }\end{array}$} & & $79,4 \%$ & $67,6 \%$ & $66,3 \%$ & $62,3 \%$ & $66,6 \%$ & $66,5 \%$ & $67,0 \%$ \\
\hline & Residuo corregido & 4,6 & 0,3 & $-0,4$ & $-2,7$ & $-0,2$ & $-0,3$ & \\
\hline \multirow{2}{*}{$\begin{array}{l}\text { No debe prohibirse ningún tipo de } \\
\text { información }\end{array}$} & & $20,2 \%$ & $30,0 \%$ & $31,5 \%$ & $35,8 \%$ & $31,8 \%$ & $30,6 \%$ & $31,0 \%$ \\
\hline & Residuo corregido & $-4,0$ & $-0,4$ & 0,3 & 2,8 & 0,5 & $-0,2$ & \\
\hline \multirow{2}{*}{ No lo sabe } & & $0,4 \%$ & $2,4 \%$ & $2,2 \%$ & $1,9 \%$ & $1,6 \%$ & $2,9 \%$ & $2,0 \%$ \\
\hline & Residuo corregido & $-2,0$ & 0,5 & 0,4 & $-0,3$ & $-0,8$ & 1,7 & \\
\hline Total & & $100 \%$ & $100 \%$ & $100 \%$ & $100 \%$ & $100 \%$ & $100 \%$ & $100 \%$ \\
\hline
\end{tabular}

Fuente: Estudio CIS n. 3279 
Por lo que respecta a la edad, la tabla 3 muestra cómo los partidarios de restringir la difusión de ciertas informaciones son especialmente los más jóvenes. Es decir, los pertenecientes a la franja de edad de entre 18 y 25 años son más propensos a querer limitar la libertad de información, con un 79,4\%, 12,4 puntos porcentuales por encima del valor total. En el otro extremo, las personas que se encuentran en el grupo de edad de entre 46 y 55 años son las que manifiestan una menor voluntad de limitar la circulación de información, con un 62,3\%. El resto de los grupos de edad no muestran desviaciones significativas con respecto a la población general. Las diferencias entre los grupos de edad mencionados son estadísticamente significativas, atendiendo a los residuos tipificados corregidos, de forma que ambas se encuentran también asociadas al 99\% de probabilidad.

Tabla 4. Actitudes hacia la prohibición de información según clase social ( $N=2.891)$

\begin{tabular}{|c|c|c|c|c|c|}
\hline & & Alta & Media & Baja & \\
\hline \multirow{2}{*}{ Habría que restringir y controlar las informaciones } & & $62,6 \%$ & $65,3 \%$ & $70,2 \%$ & $67,0 \%$ \\
\hline & Residuo corregido & $-1,8$ & $-1,8$ & 3,0 & \\
\hline \multirow{2}{*}{ No debe prohibirse ningún tipo de información } & & $36,5 \%$ & $32,6 \%$ & $27,5 \%$ & $31,0 \%$ \\
\hline & Residuo corregido & 2,3 & 1,8 & $-3,3$ & \\
\hline \multirow{2}{*}{ No lo sabe } & & $0,9 \%$ & $2,1 \%$ & $2,3 \%$ & $2,0 \%$ \\
\hline & Residuo corregido & $-1,6$ & 0,2 & 0,8 & \\
\hline Total & & $100 \%$ & $100 \%$ & $100 \%$ & $100 \%$ \\
\hline
\end{tabular}

Fuente: Estudio CIS n. 3279

En relación con la clase social a la que pertenecen los encuestados, la tabla 4 refleja cómo los individuos de clase alta son quienes más se inclinan por no controlar el flujo informativo. Por otro lado, los pertenecientes a la clase social baja son el grupo más partidario de restringir las informaciones y limitarlas a fuentes oficiales, con un 70,2\%. Así pues, las personas de clase alta se muestran más favorables a mantener la libertad de información (con un 36,5\%) en comparación con el resto, mientras las de clase baja son las menos partidarias de conservar esta libre circulación de noticias (con un porcentaje que desciende hasta el $27,5 \%$ ). Por lo que respecta a la clase media, a la que pertenece el grueso de la población, no existen diferencias significativas a tenor de los análisis estadísticos.

Tabla 5. Actitudes hacia la prohibición de la información según nivel de estudios ( $N=2961)$

\begin{tabular}{|c|c|c|c|c|c|c|c|c|}
\hline & & $\begin{array}{l}\text { Sin } \\
\text { estudios }\end{array}$ & Primaria & $\begin{array}{c}\text { Secundaria } \\
1^{\text {a }} \text { etapa }\end{array}$ & $\begin{array}{c}\text { Secundaria } \\
2^{\mathrm{a}} \text { etapa }\end{array}$ & FP & Superiores & Total \\
\hline \multirow{2}{*}{$\begin{array}{l}\text { Habría que restringir y } \\
\text { controlar las informa- } \\
\text { ciones }\end{array}$} & & $64,4 \%$ & $71,3 \%$ & $76,4 \%$ & $67,3 \%$ & $73,2 \%$ & $59,7 \%$ & $67,0 \%$ \\
\hline & Residuo corregido & $-0,4$ & 1,5 & 4,2 & 0,1 & 3,8 & $-7,0$ & \\
\hline \multirow{2}{*}{$\begin{array}{l}\text { No debe prohibirse } \\
\text { ningún tipo de infor- } \\
\text { mación }\end{array}$} & & $33,3 \%$ & $24,7 \%$ & $22,9 \%$ & $31,0 \%$ & $24,4 \%$ & $38,3 \%$ & $30,9 \%$ \\
\hline & Residuo corregido & 0,4 & $-2,2$ & $-3,7$ & 0,0 & $-4,1$ & 7,2 & \\
\hline \multirow{2}{*}{ No lo sabe } & & $2,2 \%$ & $4,0 \%$ & $0,8 \%$ & $1,7 \%$ & $2,4 \%$ & $2,0 \%$ & $2,1 \%$ \\
\hline & Residuo corregido & 0,1 & 2,2 & $-1,9$ & $-0,5$ & 0,7 & $-0,2$ & \\
\hline Total & & $100 \%$ & $100 \%$ & $100 \%$ & $100 \%$ & $100 \%$ & $100 \%$ & $100 \%$ \\
\hline
\end{tabular}

Fuente: Estudio CIS n. 3279

¿Qué sucede con el nivel de estudios? Tal como recoge la tabla 5, los con estudios de primera etapa de Secundaria son los más partidarios de limitar la información durante la pandemia del coronavirus, con un 76,4\% a favor del control. Por su parte, las personas con estudios de Formación Profesional son el segundo grupo más propenso a las restricciones a la difusión de noticias, con un $73,2 \%$. En el lado contrario, los con estudios superiores se muestran menos partidarios de la prohibición, con un $59,7 \%$. Por tanto, las personas bien con estudios en Secundaria primera etapa o bien en Formación Profesional son las más partidarias de limitar la libertad de información, mientras los con estudios superiores se inclinan más por mantener la libertad habitual en la difusión de informaciones. En todos los casos las Los individuos de clase alta son quienes más se inclinan por no controlar el flujo informativo y los de clase baja son más partidarios de restringirlo

diferencias comentadas son significativas. 
Tabla 6. Actitudes hacia la prohibición de la información según tamaño de municipio (N=2.986)

\begin{tabular}{|c|c|c|c|c|c|c|c|c|c|}
\hline & & & & & & & & \\
\hline & & $<2.000$ & $\begin{array}{l}2.001- \\
10.000\end{array}$ & $\begin{array}{c}10.001 \\
-50.000\end{array}$ & $\begin{array}{c}50.001 \\
-100.000\end{array}$ & $\begin{array}{c}100.001 \\
-400.000\end{array}$ & $\begin{array}{l}400.001- \\
1.000 .000\end{array}$ & $\begin{array}{c}>1 \\
\text { millón }\end{array}$ & \\
\hline \multirow{2}{*}{$\begin{array}{l}\text { Habría que restringir y con- } \\
\text { trolar las informaciones }\end{array}$} & & $72,8 \%$ & $72,1 \%$ & $68,3 \%$ & $66,9 \%$ & $63,8 \%$ & $61,5 \%$ & $64,1 \%$ & $67,0 \%$ \\
\hline & Residuo corregido & 1,7 & 2,5 & 0,9 & 0,0 & $-2,0$ & $-1,8$ & $-1,1$ & \\
\hline \multirow{2}{*}{$\begin{array}{l}\text { No debe prohibirse ningún } \\
\text { tipo de información }\end{array}$} & & $23,9 \%$ & $26,0 \%$ & $29,4 \%$ & $30,4 \%$ & $34,6 \%$ & $36,3 \%$ & $34,9 \%$ & $31,0 \%$ \\
\hline & Residuo corregido & $-2,1$ & $-2,4$ & $-1,1$ & $-0,3$ & 2,3 & 1,8 & 1,5 & \\
\hline \multirow{2}{*}{ No lo sabe } & & $3,3 \%$ & $1,8 \%$ & $2,3 \%$ & $2,7 \%$ & $1,6 \%$ & $2,1 \%$ & $1,0 \%$ & $2,0 \%$ \\
\hline & Residuo corregido & 1,3 & $-0,3$ & 0,6 & 0,9 & $-0,9$ & 0,1 & $-1,3$ & \\
\hline Total & & $100 \%$ & $100 \%$ & $100 \%$ & $100 \%$ & $100 \%$ & $100 \%$ & $100 \%$ & $100 \%$ \\
\hline
\end{tabular}

Fuente: Estudio CIS n. 3279

En la tabla 6 se recogen las actitudes hacia la restricción informativa según el tamaño del municipio. Las personas que viven en un municipio de menos de 2.000 habitantes son las más contrarias a la libre circulación de la información, con un $23,9 \%$, seguidas de las cuyo municipio oscila entre las 2.001 y 10.000 personas, con un $26,0 \%$. Por su parte, las personas que viven en un municipio entre 100.001 y 400.000 personas tienen mayor probabilidad de oponerse a la limitación del flujo informativo, y reflejan una mayor variación respecto al valor de la población general. El resto de categorías de municipios no ofrece desviaciones significativas con respecto a los valores de la población general.

En síntesis, los resultados en torno al perfil sociodemográfico reflejan que los partidarios de prohibir la libre circulación de la información son más habitualmente las mujeres, los jóvenes, las personas con un nivel medio de estudios, quienes pertenecen a una clase social baja, y quienes viven en municipios de pocos habitantes. Más allá del análisis de los atributos sociodemográficos, conviene interrogarse sobre el perfil ideológico de los encuestados, y analizar si las creencias políticas de los individuos están asociadas con sus actitudes en torno a la prohibición de la libertad informativa.

Tabla 7. Actitudes hacia la prohibición de la información según ideología ( $N=2.872$ )

\begin{tabular}{|c|c|c|c|c|c|c|c|}
\hline & & $\begin{array}{c}\text { Extrema } \\
\text { izquierda }\end{array}$ & Izquierda & Centro & Derecha & $\begin{array}{l}\text { Extrema } \\
\text { derecha }\end{array}$ & \\
\hline \multirow{2}{*}{$\begin{array}{l}\text { Habría que restringir y con- } \\
\text { trolar las informaciones }\end{array}$} & & $73,9 \%$ & $69,4 \%$ & $65,8 \%$ & $57,9 \%$ & $66,9 \%$ & $67,0 \%$ \\
\hline & Residuo corregido & 3,3 & 1,7 & $-1,2$ & $-3,9$ & 0,0 & \\
\hline \multirow{2}{*}{$\begin{array}{l}\text { No debe prohibirse ningún } \\
\text { tipo de información }\end{array}$} & & $24,6 \%$ & $29,2 \%$ & $31,9 \%$ & $40,2 \%$ & $30,8 \%$ & $31,2 \%$ \\
\hline & Residuo corregido & $-3,1$ & $-1,3$ & 0,8 & 4,0 & $-0,1$ & \\
\hline \multirow{2}{*}{ No lo sabe } & & $1,4 \%$ & $1,4 \%$ & $2,3 \%$ & $1,9 \%$ & $2,3 \%$ & $1,9 \%$ \\
\hline & Residuo corregido & $-0,8$ & $-1,2$ & 1,4 & 0,0 & 0,4 & \\
\hline Total & & $100 \%$ & $100 \%$ & $100 \%$ & $100 \%$ & $100 \%$ & $100 \%$ \\
\hline
\end{tabular}

Fuente: Estudio CIS n. 3279

Según la auto-ubicación ideológica, tal y como se puede observar en la tabla 7, los encuestados que se consideran de extrema izquierda (1-2) son más a menudo favorables a la restricción del flujo informativo, con un 73,9\%, 6,9 puntos por encima del valor de la población general. Por su parte, el 57,9\% de los que se ubican a la derecha son partidarios de este control, 9,9 puntos menos que el total, lo que los convierte en el grupo ideológico que menos apoya las medidas coercitivas. Es decir, los únicos grupos ideológicos para los que se observan desviaciones significativas en el porcentaje de apoyo a la restricción informativa con respecto a la población general son la extrema izquierda y la derecha. El resto de los segmentos ideológicos de la población mantiene valores similares al de la población general. Podemos afirmar que los que se consideran de extrema izquierda tienen una mayor probabilidad de ser partidarios de limitar la información durante la crisis sanitaria, mientras que las personas que se sitúan en la derecha son menos proclives.

Pero ¿se traducen estas diferencias ideológicas observadas en el comportamiento electoral? Es decir, ¿'hallamos también diferencias en las actitudes hacia la prohibición en función del partido político votado por los individuos en las últimas elecciones generales?

Los partidarios de prohibir la libre circulación de la información son más habitualmente mujeres, jóvenes, personas con nivel medio de estudios, de clase social baja y quienes viven en municipios de pocos habitantes 
Tabla 8. Actitudes hacia la prohibición de la información según recuerdo de voto en las elecciones generales del 10 de noviembre de 2019 (N=2.161)

\begin{tabular}{|c|c|c|c|c|c|c|c|c|}
\hline & \multirow{3}{*}{$\begin{array}{c}\boldsymbol{P P} \\
57,6 \%\end{array}$} & \multirow{3}{*}{$\begin{array}{l}\text { PSOE } \\
76,4 \%\end{array}$} & \multirow{3}{*}{$\begin{array}{c}\text { UP } \\
67,3 \%\end{array}$} & \multirow{3}{*}{$\frac{\text { Cs }}{62,4 \%}$} & \multirow{3}{*}{$\begin{array}{c}\text { Vox } \\
56,7 \%\end{array}$} & \multirow{3}{*}{$\begin{array}{l}\text { Otros } \\
65,0 \%\end{array}$} & \multirow[b]{3}{*}{$67,0 \%$} \\
\hline & & & & & & & & \\
\hline Habría que restringir y con- & & & & & & & & \\
\hline trolar las informaciones & Residuo corregido & $-4,3$ & 6,8 & 0,1 & $-1,5$ & $-3,2$ & $-0,8$ & \\
\hline \multirow{2}{*}{$\begin{array}{l}\text { No debe prohibirse ningún } \\
\text { tipo de información }\end{array}$} & & $40,1 \%$ & $22,2 \%$ & $31,4 \%$ & $35,7 \%$ & $42,3 \%$ & $33,0 \%$ & $31,4 \%$ \\
\hline & Residuo corregido & 4,1 & $-6,8$ & 0,0 & 1,4 & 3,5 & 0,7 & \\
\hline \multirow{2}{*}{ No lo sabe } & & $2,3 \%$ & $1,5 \%$ & $1,3 \%$ & $1,9 \%$ & $1,0 \%$ & $2,0 \%$ & $1,7 \%$ \\
\hline & Residuo corregido & 1,1 & $-0,6$ & $-0,5$ & 0,3 & $-0,8$ & 0,5 & \\
\hline Total & & $100 \%$ & $100 \%$ & $100 \%$ & $100 \%$ & $100 \%$ & $100 \%$ & $100 \%$ \\
\hline
\end{tabular}

Fuente: Estudio CIS n. 3279

Según el comportamiento electoral, los votantes del PSOE tienen la mayor probabilidad de estar a favor de la aplicación de medidas que limiten la libertad de información relativa a la pandemia, con un 76,4\%. Por su parte, los votantes del PP y de Vox son significativamente menos partidarios de controlar las informaciones, con porcentajes similares (tabla 8). El resto de los votantes de otros partidos (Unidas Podemos, Ciudadanos y otros) no exhibe diferencias significativas con respecto a las respuestas de la población general.

Estos resultados ponen de manifiesto que las actitudes en torno a la libertad de la informativa están asociadas no sólo a atributos sociodemográficas (sexo, edad, nivel de estudios, tamaño de municipio y clase social), sino también a las actitudes políticas de los individuos -en relación con el Gobierno de coalición-, tanto en lo que respecta a sus orientaciones ideológicas generales, como a su preferencia específica por determinados partidos. Así lo refleja con rotundidad el hecho de que las desviaciones más importantes se localicen por una parte en la extrema izquierda y los votantes del $P S O E$, que son quienes muestran un apoyo más unánime a la restricción de la información, y por otra, en la derecha y los votantes de Vox y del $P P$, que son quienes más intensamente criticaron la gestión gubernamental de la crisis sanitaria de la Covid-19, y más se opusieron a la limitación de la libertad de información.

Tabla 9. Actitudes hacia la prohibición de la información según simpatía por partidos políticos ( $\mathrm{N}=387$ )

\begin{tabular}{|c|c|c|c|c|c|c|c|c|}
\hline & \multirow{3}{*}{$\begin{array}{c}P \boldsymbol{P} \\
66,2 \%\end{array}$} & \multirow{3}{*}{$\begin{array}{c}\text { PSOE } \\
81,0 \%\end{array}$} & \multirow{3}{*}{$\begin{array}{c}\boldsymbol{U P} \\
61,1 \%\end{array}$} & & & & \\
\hline & & & & & \multirow{2}{*}{$\begin{array}{c}\text { Cs } \\
59,3 \%\end{array}$} & \multirow{2}{*}{$\begin{array}{c}\text { Vox } \\
41,7 \%\end{array}$} & \multirow{2}{*}{$\begin{array}{l}\text { Otros } \\
61,5 \%\end{array}$} & \multirow{2}{*}{$\begin{array}{l}\text { Total } \\
68,7 \%\end{array}$} \\
\hline Habría que restringir y contro- & & & & & & & & \\
\hline lar las informaciones & Residuo corregido & $-0,5$ & 4,1 & $-1,0$ & $-1,7$ & $-2,1$ & $-1,4$ & \\
\hline \multirow{2}{*}{$\begin{array}{l}\text { No debe prohibirse ningún } \\
\text { tipo de información }\end{array}$} & & $30,9 \%$ & $18,4 \%$ & $36,1 \%$ & $39,0 \%$ & $58,3 \%$ & $36,9 \%$ & $29,7 \%$ \\
\hline & Residuo corregido & 0,2 & $-3,8$ & 0,9 & 1,7 & 2,2 & 1,4 & \\
\hline \multirow{2}{*}{ No lo sabe } & & $2,9 \%$ & $0,7 \%$ & $2,8 \%$ & $1,7 \%$ & $0,0 \%$ & $1,5 \%$ & $1,6 \%$ \\
\hline & Residuo corregido & 1,0 & $-1,1$ & 0,6 & 0,1 & $-0,4$ & 0,0 & \\
\hline Total & & $100 \%$ & $100 \%$ & $100 \%$ & $100 \%$ & $100 \%$ & $100 \%$ & $100 \%$ \\
\hline
\end{tabular}

Fuente: Estudio CIS n. 3279

Además, con el fin de confirmar los hallazgos anteriores, hemos cruzado la simpatía por el partido político con la polémica pregunta del CIS en torno a la prohibición de la información. Esta pregunta es una herramienta clásica del CIS para tratar de ubicar ideológica y políticamente a muchos encuestados que no revelan su voto en las preguntas relativas al comportamiento electoral, así como para intentar realizar estimaciones de intención de voto en los estudios preelectorales. Los resultados revelan que los encuestados que simpatizan con el PSOE son significativamente más favorables a la limitación de la información, lo que confirma los resultados hallados en relación con el recuerdo de voto (tabla 8). Además, en este caso el porcentaje es notablemente más alto y se incrementa hasta alcanzar el $81,0 \%$, lo que refleja diferencias más significativas, con un $99 \%$ de probabilidad. Los simpatizantes de Vox por su parte, son los menos partidarios del control de las informaciones durante la crisis sanitaria de la Covid-19, con únicamente el $41,7 \%$ a favor, en este caso al $95 \%$ de probabilidad. De esta manera, se convierten en el único partido político cuyos simpatizantes son más propensos a no limitar el flujo de noticias e informaciones.

Los dos grupos de población más favorables al control informativo son quienes manifiestan mucha o bastante confianza en la actuación del Gobierno con motivo de la crisis sanitaria 
Tabla 10. Actitudes hacia la prohibición de la información según confianza en la política del Gobierno central contra la Covid-19 (N=2.957)

\begin{tabular}{|c|c|c|c|c|c|c|c|}
\hline & \multirow{2}{*}{$\begin{array}{c}\text { Mucha } \\
73,8 \%\end{array}$} & \multirow{2}{*}{$\begin{array}{c}\text { Bastante } \\
72,5 \%\end{array}$} & \multirow{2}{*}{$\begin{array}{c}\text { Regular } \\
61,5 \%\end{array}$} & \multirow{2}{*}{$\begin{array}{c}\text { Poca } \\
67,2 \%\end{array}$} & \multirow{2}{*}{$\begin{array}{c}\text { Ninguna } \\
52,8 \%\end{array}$} & \multirow{2}{*}{$\begin{array}{l}\text { Total } \\
67,0 \%\end{array}$} \\
\hline Habría que restringir y controlar & & & & & & & \\
\hline las informaciones & Residuo corregido & 2,7 & 4,9 & $-1,4$ & 0,2 & $-7,7$ & \\
\hline \multirow{2}{*}{$\begin{array}{l}\text { No debe prohibirse ningún tipo } \\
\text { de información }\end{array}$} & & $24,3 \%$ & $25,6 \%$ & $32,9 \%$ & $31,0 \%$ & $45,3 \%$ & $31,0 \%$ \\
\hline & Residuo corregido & $-2,7$ & $-4,8$ & 0,5 & 0,0 & 7,9 & \\
\hline \multirow{2}{*}{ No lo sabe } & & $1,9 \%$ & $1,9 \%$ & $5,6 \%$ & $1,8 \%$ & $1,9 \%$ & $2,0 \%$ \\
\hline & Residuo corregido & $-0,1$ & $-0,5$ & 3,1 & $-0,6$ & $-0,3$ & \\
\hline Total & & $100 \%$ & $100 \%$ & $100 \%$ & $100 \%$ & $100 \%$ & $100 \%$ \\
\hline
\end{tabular}

Fuente: Estudio CIS n. 3279

Para finalizar, hemos cruzado la pregunta en cuestión con dos últimas variables relativas a la confianza en la actuación del Gobierno central con respecto a la pandemia por una parte, y a la confianza en la figura del presidente del Gobierno por otra (tablas 10 y 11). Los resultados reflejan que existe asociación estadística en ambos casos, y que la confianza tanto en el Gobierno central en su conjunto como en la figura particular del presidente del Gobierno, Pedro Sánchez, se asocia con una mayor probabilidad de apoyar la restricción de las informaciones durante la pandemia.

Así, quienes manifiestan mucha o bastante confianza en la actuación del Gobierno con motivo de la crisis sanitaria son los dos grupos de población más favorables al control informativo (un 73,8\% de los primeros y un $72,5 \%$ de los segundos lo apoyan, frente al $67,7 \%$ de la población general). La misma tendencia se observa en la dirección opuesta, en quienes no exhiben ninguna confianza en la política del Gobierno central, que tienen una probabilidad significativamente menor de apoyar la restricción del flujo informativo (52,8\% vs. 67 \%). Las personas que tienen poca o regular confianza en la gestión del Gobierno socialista no exhiben desviación con respecto a la población general. Los resultados confirman de nuevo que las actitudes políticas hacia el Gobierno central están claramente asociadas con la opinión de los encuestados sobre la pertinencia de limitar el flujo informativo.

Tabla 11. Actitudes hacia la prohibición de la información según confianza en el presidente del Gobierno (N=2.957)

\begin{tabular}{|c|c|c|c|c|c|c|}
\hline & & Mucha & Bastante & Poca & Ninguna & Total \\
\hline \multirow{2}{*}{$\begin{array}{l}\text { Habría que restringir y controlar las } \\
\text { informaciones }\end{array}$} & & $76,1 \%$ & $73,7 \%$ & $68,1 \%$ & $55,4 \%$ & $66,9 \%$ \\
\hline & Residuo corregido & 3,0 & 5,4 & 1,0 & $-8,3$ & \\
\hline \multirow{2}{*}{$\begin{array}{l}\text { No debe prohibirse ningún tipo de } \\
\text { información }\end{array}$} & & $22,1 \%$ & $24,4 \%$ & $29,6 \%$ & $42,8 \%$ & $31,1 \%$ \\
\hline & Residuo corregido & $-3,0$ & $-5,4$ & $-1,2$ & 8,6 & \\
\hline \multirow{2}{*}{ No lo sabe } & & $1,8 \%$ & $1,9 \%$ & $2,3 \%$ & $1,8 \%$ & $2,0 \%$ \\
\hline & Residuo corregido & $-0,2$ & $-0,2$ & 0,8 & $-0,5$ & \\
\hline Total & & $100 \%$ & $100 \%$ & $100 \%$ & $100 \%$ & $100 \%$ \\
\hline
\end{tabular}

Fuente: Estudio CIS n. 3279

Con respecto a la confianza en el jefe del Ejecutivo, se observa la misma tendencia. Quienes tienen mucha o bastante confianza, son significativamente más partidarios de la restricción informativa, y quienes no tienen ninguna confianza en Pedro Sánchez, son mucho menos favorables al control informativo. En síntesis, los resultados ponen de manifiesto que tener confianza en la gestión del Gobierno y en la figura del presidente está significativamente asociado con tener mayor inclinación de restringir la información, tal como sugería el propio Gobierno.

\section{Conclusiones}

Conviene sintetizar los resultados de esta investigación y responder a los objetivos que la han guiado. Con motivo del primer objetivo (01), analizar las características sociodemográficas de quienes se declararon partidarios de limitar la libertad de información durante el Estado de alarma, los resultados señalan que las mujeres fueron más partidarias de la prohibición del flujo informativo que los hombres. Además, los jóvenes entre 18 y 25 años fueron el grupo de edad con más probabilidad de apoyar la restricción, junto a las personas que se auto-ubicaron en la clase social baja. Respecto a la educación, los más proclives a la prohibición fueron los con estudios secundarios -bien de primera etapa o bien de formación profesional-. Por último, en cuanto al tamaño del municipio, fueron más partidarias de restringir la libertad de información las personas que vivían en municipios por debajo de los 10.000 habitantes.

Por el contrario, el perfil sociodemográfico de quienes prefirieron mantener la libertad de información sin restricciones de ningún tipo se corresponde con varones de edad adulta -de entre 46 y 55 años- con estudios superiores y ubicados en una clase social alta. En cuanto al municipio, los habitantes de ciudades de tamaño medio -municipios de entre 100.000 y 400.00 habitantes- fueron los más partidarios de la libre circulación de información. 
Con respecto al segundo objetivo (02), analizar las actitudes políticas y el perfil ideológico de quienes declararon estar a favor de limitar el flujo informativo, las preferencias ideológicas y políticas generales difieren significativamente entre ambos grupos de población (prohibicionista vs. no prohibicionistas), y están estadísticamente asociadas con la opinión sobre la libertad de información.

En primer lugar, los más proclives a la prohibición de la libre circulación informativa se ubicaron más a menudo en la extrema izquierda y además eran con mayor frecuencia votantes y simpatizantes del principal partido del Gobierno: el PSOE. Esto no sucedió en cambio con el otro partido que conforma el Gobierno, pues los resultados no reflejan que los simpatizantes y votantes de Podemos fueran significativamente más partidarios de la restricción de la libre circulación de noticias. En segundo lugar, los partidarios de la restricción informativa tenían mucha o bastante confianza tanto en la gestión de la pandemia por parte del Gobierno central como en la figura del presidente del Gobierno.

Por su parte, los partidarios de mantener la libertad de información se ubicaron más a menudo en la derecha y eran con mayor frecuencia votantes y/o simpatizantes del PP y/o Vox. Además, no confiaron en la gestión gubernamental del Gobierno de coalición de la pandemia y no tenían ninguna confianza en el presidente del Gobierno. En otras palabras, las actitudes ideológicas y políticas se asociaron de forma significativa con las actitudes que la población expresó en torno a la necesidad de restringir la libertad de información.

¿Cómo cabe interpretar estos resultados? ¿Por qué hallamos esta asociación sistemática entre las actitudes políticas e ideológicas generales de la población y las actitudes en torno a una cuestión tan específica como la posibilidad de restringir el flujo informativo? Además, resulta llamativo que la asociación estadística no se produzca, como cabría esperar, entre actitudes políticas más próximas al autoritarismo y la inclinación por la prohibición. La asociación adopta por el contrario una forma más interesante: se produce entre las actitudes políticas de la ciudadanía y las posturas adoptadas públicamente por los partidos políticos españoles sobre el asunto de la libertad de información.

La respuesta tentativa es que la gente utilizó sus actitudes políticas básicas -identificación partidista y auto-ubicación ideológica- como heurísticas o atajos cognitivos para tomar posición en torno a la cuestión de la restricción de la libertad de información. Es decir, buena parte de la población hizo suya la postura adoptada públicamente por los partidos políticos de referencia con los que simpatizaba. Así, quienes apoyaban al Gobierno de coalición (especialmente al PSOE) tuvieron una mayor probabilidad de apoyar las medidas sugeridas por éste, incluso aunque ello supusiera la limitación inusual de un derecho (a la información) que la izquierda siempre ha juzgado fundamental para el correcto funcionamiento de la democracia. Y lo hicieron probablemente como resultado de las diversas declaraciones públicas de varios ministros en relación con los peligros de los bulos, la desinformación, etc. y la necesidad de limitar su circulación en plena crisis sanitaria. Por su parte, quienes simpatizaban con el PP o Vox tuvieron una mayor probabilidad de preferir mantener la libertad de información, en línea con las posturas adoptadas respectivamente por ambas fuerzas políticas, que criticaron duramente al Gobierno por sus coqueteos con la idea de restringir la libertad informativa.

La importancia de la identificación partidista y la auto-ubicación ideológica como heurísticas o atajos cognitivos que guían la formación de opiniones y el comportamiento político de los ciudadanos ha sido abundantemente teorizada y acreditada por estudios empíricos procedentes del ámbito de la ciencia política (Lodge; Hamill, 1986; Conover; Feldman, 1989; Lau; Redlawsk, 2001). Su centralidad es tan relevante que los estudios en comunicación constatan también que ambas son a menudo utilizadas como guías del comportamiento mediático, es decir, motivan la selección de medios de comunicación y contenidos que coincidan con las actitudes políticas básicas de los individuos (Stroud, 2008; 2011; Humanes, 2014, Humanes; Mellado, 2017).

Según la célebre perspectiva de los "avaros cognitivos" procedente de la psicología cognitiva (Kahneman; Slovic; Tversky, 1982), utilizamos atajos constantemente para formarnos imágenes mentales del entorno que nos rodea y tomar decisiones eficientes con el mínimo esfuerzo mental en diferentes aspectos de nuestra vida, como ya planteaba en la década de los años 20 de forma brillante Lippmann (1922). Así, el presente estudio sugiere que la ideología y la identificación/simpatía partidista actuaron como claves cognitivas que facilitaron a los ciudadanos españoles la toma de postura en torno a la cuestión de la libertad de información en un contexto de gran incertidumbre y demanda de información.

De esta forma, los resultados a la pregunta del barómetro del CIS no deben entenderse únicamente como el resultado del miedo a la desinformación y el caos informativo en el contexto de la crisis sanitaria, sino como un resultado influido por el hecho de que los ciudadanos utilizaron sus actitudes políticas básicas como estándares para evaluar si estaban o no a favor de restringir el flujo informativo, en función de su adscripción a las dos esferas ideológicas de referencia. Así, no es que España se acostara enemiga de limitar la libertad de información y se levantara súbitamente prohibicionista, sólo siguió siendo significativamente partidista.
La ideología y la identificación/simpatía partidista actuaron como claves cognitivas que facilitaron a los ciudadanos españoles su toma de postura 


\section{Referencias}

Aleixandre-Benavent, Rafael; Castelló-Cogollos, Lourdes; Valderrama-Zurián, Juan-Carlos (2020). “Información y comunicación durante los primeros meses de Covid-19. Infodemia, desinformación y papel de los profesionales de la información". Profesional de la información, v. 29, n. 4, e290408.

https://doi.org/10.3145/epi.2020.jul.08

Amat, Francesc; Falcó-Gimeno, Albert; Arenas, Andreu; Muñoz, Jordi (2020). “Pandemics meet democracy. Experimental evidence from the Covid-19 crisis in Spain". SocArXiv, 6 April.

https://doi.org/10.31235/osf.io/dkusw

Arias-Maldonado, Manuel (2016). La democracia sentimental: política y emociones en el siglo XXI. Barcelona: Página Indómita. ISBN: 9788494481659

Bennett, W. Lance; lyengar, Shanto (2008). "A new era of minimal effects? The changing foundations of political communication". Journal of communication, v. 58, n. 4, pp. 707-731.

https://doi.org/10.1111/j.1460-2466.2008.00410.x

Broersma, Marcel; Graham, Todd (2013). "Twitter as a news source”. Journalism practice, v. 7, n. 4, pp. $446-464$. https://doi.org/10.1080/17512786.2013.802481

Bos, Linda; Kruikemeier, Sanne; De-Vreese, Claes (2016). “Nation binding: How public service broadcasting mitigates political selective exposure". PloS one, v. 11, n. 5, e0155112.

https://doi.org/10.1371/journal.pone.0155112

Carratalá, Adolfo; Valera-Ordaz, Lidia (2020). "El pluralismo polarizado ante la nueva política y el columnismo digital”. Estudios sobre el mensaje periodístico, v. 26, n. 1, pp. 71-83.

https://doi.org/10.5209/esmp.67287

Casero-Ripollés, Andreu (2020). "Impact of Covid-19 on the media system. Communicative and democratic consequences of news consumption during the outbreak". El profesional de la información, v. 29, n. 2, e290223.

https://doi.org/10.3145/epi.2020.mar.23

Chadwick, Andrew (2013). The hybrid media system: Politics and power. Oxford: Oxford University Press. ISBN: 9780 190696733

Conover, Pamela J.; Feldman, Stanley (1989). "Candidate perception in an ambiguous world: Campaigns, cues, and inference processes". American journal of political science, v. 33, n. 4, pp. 912-940.

https://doi.org/10.2307/2111115

Cooke, Nicole A. (2018). Fake news and alternative facts: Information literacy in a post-truth era. Chicago: ALA Editions. ISBN: 9780838916360

Drylie-Carey, Lindsey; Sánchez-Castillo, Sebastián; Galán-Cubillo, Esteban (2020). “European leaders unmasked: Covid-19 communication strategy through Twitter". Profesional de la información, v. 29, n. 5, e290504.

https://doi.org/10.3145/epi.2020.sep.04

España (1978). “Constitución española”. BOE, n. 311, 29 diciembre, pp. 29313-29424.

https://www.boe.es/buscar/act.php?id=BOE-A-1978-31229

European Commission (2018a). Comunicación de la Comisión al Parlamento Europeo, al Consejo, al Comité Económico y Social Europeo y al Comité de las Regiones "La lucha contra la desinformación: un enfoque europeo". COM/2018/236final. https://eur-lex.europa.eu/legal-content/es/TXT/?uri=CELEX\%3A52018DC0236

European Commission (2018b). Code of practice on disinformation. Shaping Europe's digital future. https://ec.europa.eu/digital-single-market/en/news/code-practice-disinformation

Fisher, Caroline; Culloty, Eileen; Lee, Jee-Young; Park, Sora (2019). "Regaining control citizens who follow politicians on social media and their perceptions of journalism". Digital journalism, v. 7, n. 2, pp. 230-250.

https://doi.org/10.1080/21670811.2018.1519375

Goldman, Seth K.; Mutz, Diana C. (2011). "The friendly media phenomenon: A cross national analysis of cross-cutting exposure". Political communication, v. 28, n. 1, pp. 42-66.

https://doi.org/10.1080/10584609.2010.544280

Graves, Lucas (2017). "Anatomy of a fact check: Objective practice and the contested epistemology of fact checking". Communication, culture \& critique, v. 10, n. 3, pp. 518-537.

https://doi.org/10.1111/cccr.12163

Hallin, Daniel C.; Mancini, Paolo (2004). Comparing media systems: Three models of media and politics. Cambridge: Cambridge University Press. ISBN: 9780521543088 
Hameleers, Michael; Van-der-Meer, Toni G. L. A. (2019). “Misinformation and polarization in a high-choice media environment: How effective are political fact-checkers?". Communication research, v. 47, n. 2, pp. 227-250.

https://doi.org/10.1177/0093650218819671

Humanes, María-Luisa (2014). “Exposición selectiva y partidismo de las audiencias en España. El consumo de información política durante las campañas electorales de 2008". Palabra clave, v. 17, n. 3, pp. 773-802.

https://doi.org/10.5294/pacla.2014.17.3.9

Humanes, María-Luisa; Mellado, Claudia (2017). “Modelos explicativos de la exposición selectiva a la información política y partidismo de las audiencias en España". In: IAMCR Conference transforming culture, politics \& communication: New media, new territories, new discourses". Cartagena de Indias, 16-20 July 2017.

https://www.researchgate.net/publication/318323608_Modelos_explicativos_de_la_exposicion_selectiva_a_la_ informacion_politica_y_partidismo_de_las_audiencias_en_Espana

Kahneman, Daniel; Slovic, Paul; Tversky, Amos (eds.) (1982). Judgment under uncertainty: Heuristics and biases. Cambridge University Press. ISBN: 9780521284141

Lau, Richard R.; Redlawsk, David P. (2001). "Advantages and disadvantages of cognitive heuristics in political decision making". American journal of political science, v. 45, n. 4, pp. 951-971.

https://doi.org/10.2307/2669334

Lippmann, Walter (1922). Public opinion. Harcourt. ISBN: 9780029191309

Lodge, Milton; Hamill, Ruth (1986). "A partisan schema for political information processing”. American political science review, v. 80, n. 2, pp. 505-519.

https://doi.org/10.2307/1958271

López-García, Guillermo (2020). "Vigilar y castigar: el papel de militares, policías y guardias civiles en la comunicación de la crisis del Covid-19 en España”. El profesional de la información, v. 29, n. 3, e290311.

https://doi.org/10.3145/epi.2020.may.11

López-Pan, Fernando; Rodríguez-Rodríguez; Jorge-Miguel (2020). “El fact checking en España. Plataformas, prácticas y rasgos distintivos". Estudios sobre el mensaje periodístico, v. 26, n. 3, pp. 1045-1065

https://doi.org/10.5209/esmp.65246

Masip, Pere; Ruiz-Caballero, Carlos; Suau, Jaume (2019). “Active audiences and social discussion on the digital public sphere. Review article". El profesional de la información, v. 28, n. 2, e280204.

https://doi.org//10.3145/epi.2019.mar.04

Moreno, Ángeles; Fuentes-Lara, Cristina; Navarro, Cristina (2020). "Covid-19 communication management in Spain: Exploring the effect of information-seeking behavior and message reception in public's evaluation". Profesional de la información, v. 29, n. 4, e290402.

https://doi.org/10.3145/epi.2020.jul.02

Mullainathan, Senghil; Shleifer, Andrei (2005). "The market for news". American economic review, v. 95, pp. 1031-1053. https://doi.org/10.1257/0002828054825619

Organización de Naciones Unidas (2017). Declaración conjunta sobre libertad de expresión y noticias falsas ('fake news'), desinformación y propaganda.

http://www.oas.org/es/cidh/expresion/showarticle.asp?art/D=1056\&/ID=2

Paniagua-Rojano, Francisco; Seoane-Pérez, Francisco; Magallón-Rosa, Raúl (2020). “Anatomía del bulo electoral: la desinformación política durante la campaña del 28-A en España". Revista Cidob d'afers internacionals, n. 124, pp. 123-145. https://doi.org/10.24241/rcai.2020.124.1.123

Rosenberg, Hans; Syed, Shahbaz; Rezaie, Salim (2020). "The Twitter pandemic: The critical role of Twitter in the dissemination of medical information and misinformation during the Covid-19 pandemic". Canadian journal of emergency medicine, v. 22, n. 4, pp. 418-421.

https://doi.org/10.1017/cem.2020.361

Shin, Jieun; Thorson, Kjerstin (2017). "Partisan selective sharing: The biased diffusion of fact-checking messages on social media". Journal of communication, v. 67, n. 2, pp. 233-255.

https://doi.org/10.1111/jcom.12284

Stroud, Natalie-Jomini (2008). "Media use and political predispositions: Revisiting the concept of selective exposure". Political communication, v. 30, n. 3, pp. 341-366.

https://doi.org/10.1007/s11109-007-9050-9 
Stroud, Natalie-Jomini (2010). “Polarization and partisan selective exposure”. Journal of communication, v. 60, n. 3, pp. 556-576.

https://doi.org/10.1111/j.1460-2466.2010.01497.x

Stroud, Natalie-Jomini (2011). Niche news: The politics of news choice. Oxford: Oxford University Press. ISBN: 9780 199755516

Valera-Ordaz, Lidia (2015). “Building the news media agenda: Spanish political parties' influence on traditional media and blogosphere agendas during 2011's electoral campaign". Communication \& society, v. 28, n. 3, pp. 115-136.

https://doi.org/10.15581/003.28.3.115-134

Valera-Ordaz, Lidia (2019). "Frame building and frame sponsorship in the 2011 Spanish election: The practices of polarised pluralism". Contemporary social science, v. 14, n. 1, pp. 114-131.

https://doi.org/10.1080/21582041.2017.1347703

Van Aelst, Peter; Strömbäck, Jesper; Aalberg, Toril; Esser, Frank; de Vreese, Claes; Matthes, Jörg; Hopmann, David; Salgado, Susana; Hubé, Nicolas; Stepinska, Agnieszka; Papathanassopoulos, Stylianos; Berganza, Rosa; Legante, Guido; Reinemann, Carsten; Sheafer, Tamir; Stanyer, James (2017). "Political communication in a high-choice media environment: a challenge for democracy?". Annals of the International Communication Association, v. 41, n. 1, pp. 3-27.

https://doi.org/10.1080/23808985.2017.1288551

Van-Dalen, Arjen; De-Vreese, Claes H.; Albæk, Erik (2012). “Different roles, different content? A four-country comparison of the role conceptions and reporting style of political journalists". Journalism, v. 13, n. 7, pp. 903-922. https://doi.org/10.1177/1464884911431538

Villena-Alarcón, Eduardo; Caballero-Galeote, Lidia (2020). "Covid-9 media coverage on Spanish public TV". Trípodos, v. 2, n. 47, pp. 103-125.

http://www.tripodos.com/index.php/Facultat_Comunicacio_Blanquerna/article/view/818

\section{Realiza tu proyecto}

Por una biblioteca pública social e inclusiva

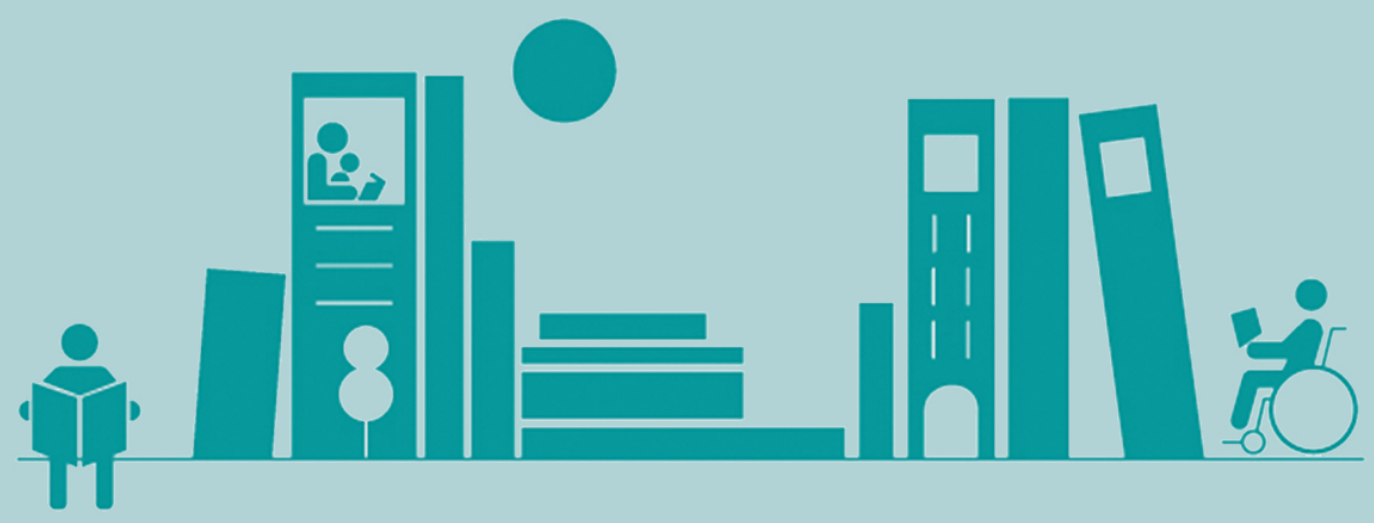

En nuestra web te ofrecemos unas pautas

para facilitar la formulación de tu proyecto fundacionbibliotecasocial.org

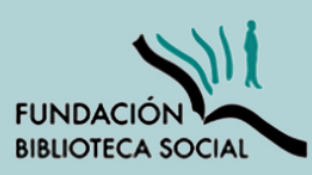

info@fundacionbibliotecasocial.org Y@Biblio_Social

f FundacionBibliotecasSocial

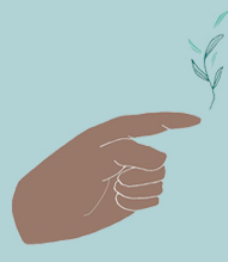

Hazte amigo/amiga de la Fundación 